\title{
ILLUMINATION FROM A RADIATING DISK
}

\author{
By Paul D. Foote, Assistant Physicist
}

Some time ago there appeared in the transactions of the Illuminating Engineering Society a discussion of the illumination produced by a diffusely and uniformly radiating circular disk at any point in space on a surface parallel to the disk. ${ }^{1}$

The integral there obtained is incorrect and the approximation method given in detail is not warranted, since the correct solution is very readily obtained and is much simpler and more convenient to use than the approximation formula presented in the paper above referred to. Very possibly the solution of this problem has appeared in various journals, but it seemed worth while to call attention to the question at the present time.

\section{REFERRING TO FIG. 1}

Problem: To find the illumination $E$ due to a circular disk of uniform brightness $i$ and radius $a$, at any point in space on a surface parallel to the disk.

Assumptions: (I) Diffuse radiation and hence the validity of Lambert's cosine law.

(2) Inverse square law for infinitesimal points.

Notation: $O C=$ perpendicular to the disk at its center.

$D=$ point at which the illumination is desired.

$X=$ perpendicular from $D$ to line $O C$, i. e. $=$ distance of $D$ from axis of symmetry.

$K=$ perpendicular distance of $D$ from plane of disk. Other quantities are evident from Fig. I. 
$E=\frac{i d w \cos ^{2} \theta}{r^{2}}$ due to element $d w$ at $B$.

$$
=i K^{2} \int_{0}^{a} \int_{0}^{2 \pi} \frac{\rho d \psi d \rho}{\left(x^{2}+\rho^{2}+K^{2}-2 x \rho \sin \psi\right)^{2}}
$$

Put $\cos \alpha=\sin \psi$ and integrate in respect to $d \alpha$.

Let $P=x^{2}+\rho^{2}+K^{2}$.

$$
Q=-2 x \rho
$$

$E=i K^{2} \int_{0}^{a} \rho d \rho \int_{0}^{2 \pi} \frac{d \alpha}{(P+Q \cos \alpha)^{2}}$

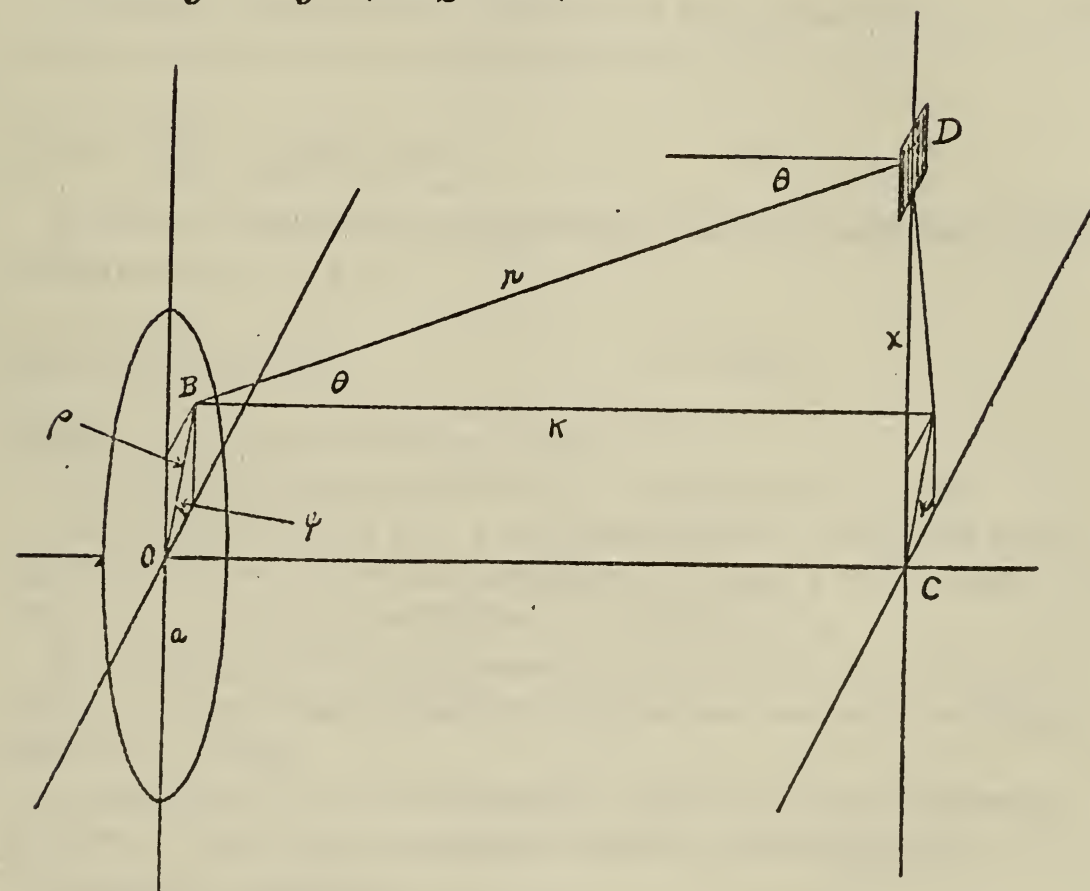

FIG. I

$P^{2}$ must be greater than $Q^{2}$ for any possible combination of variables or constants, if $K>0$ when $x<a$. Hence:

$$
\begin{aligned}
& E=2 \pi i K^{2} \int_{0}^{a} \frac{P \rho d \rho}{\left(P^{2}-Q^{2}\right)^{r}} \quad K>0 \text { when } x<a \\
& \text { Put } A=x^{2}+K^{2} \\
& B=-2\left(x^{2}-K^{2}\right) \\
& \text { Let } y=\rho^{2}
\end{aligned}
$$


Then:

$$
E=A \pi i K^{2} \int_{0}^{a^{2}} \frac{d y}{\left(A^{2}+B y+y^{2}\right)^{i}}+\pi i K^{2} \int_{0}^{a^{2}} \frac{y d y}{\left(A^{2}+B y+y^{2}\right)^{1}}
$$

(1) $E=\frac{\pi}{2}\left[1-\frac{x^{2}+K^{2}-a^{2}}{\sqrt{x^{4}+2\left(K^{2}-a^{2}\right) x^{2}+\left(K^{2}+a^{2}\right)^{2}}}\right]=$

illumination at any point in space on surface parallel to the disk, except $K=o$ when $x<a$.

\section{SPECIAL CASES}

Problem: Illumination at point on a line perpendicular to disk from a point on the circumference of the disk, i. e., $x=a$.
(2) $E=\frac{\pi i}{2}\left[1-\frac{K}{\sqrt{K^{2}+4 a^{2}}}\right]$
$K \neq 0$

Problem: Illumination at point on a line perpendicular to disk at the center, i. e., $x=0$.
(3) $E=\frac{\pi i}{2}\left[\frac{2 a^{2}}{K^{2}+a^{2}}\right]$
$K \neq 0$

which is the form clerived by Hyde. ${ }^{2}$

As a particular example, let $\pi i / 2=1$ unit, and $a=1$ unit.

For equation (I) let $x=1$.I units and let it be desired to obtain the illumination at various distances $K$ along a line parallel to the axis of symmetry but distant I.I units from it.

By equation (2) the illumination is obtained at various distances $K$ along a line parallel to the axis of symmetry but distant from it by I unit.

By equation (3) the illumination along the axis of symmetry is given. These three equations take the following form for the above special conditions.
(I) $E^{\prime}=\left[\mathrm{I}-\frac{K^{2}+0.21}{\sqrt{K^{4}+4 \cdot 4^{2} K^{2}+0.044^{1}}}\right]$
(no restriction on $K$ )
(2) $E^{\prime}=\left[1-\frac{K}{\sqrt{K^{2}+4}}\right]$
$K \neq 0$
(3) $E^{\prime}=\frac{2}{K^{2}+1}$
$K \neq o$ 
These three curves are represented graphically by Fig. 2. The general equation derived above does not hold for $K=0$ when $x<a$, but is correct for any distance greater than zero, even though this distance be infinitely small. The illumination approaches,

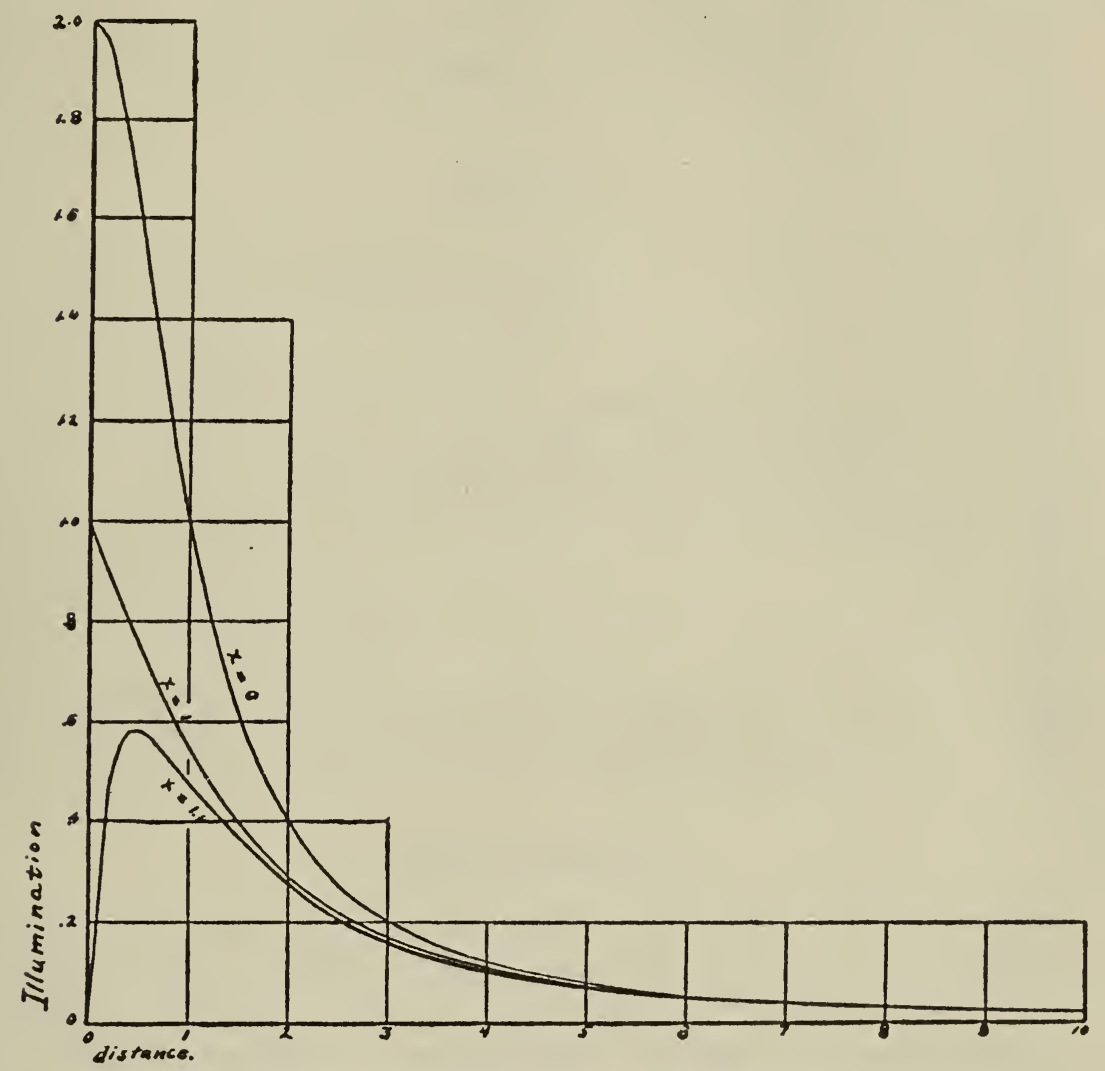

FIG. 2.-Illumination versus distance from diffusely radiating disk

for example, when $x=\mathrm{I}$, the value of $\mathrm{I}$, as $K$ approaches $o$, but it is conceivable that the illumination may have a different value at the mathematical point $K=0$.

Acknowledgment is due Dr. Olshausen, of this Bureau, for critically reading this manuscript and making suggestions.

WASHINGTON, October 30, 1915. 